\title{
Contrast enhancement methods in sodium MR imaging: a new emerging technique
}

\author{
Rakesh Sharma ${ }^{1}$, Avdhesh Sharma ${ }^{2}$, Soonjo Kwon ${ }^{3}$, Ross Booth ${ }^{3}$ \\ ${ }^{1}$ Department of Medicine, Columbia University, New York, USA; ${ }^{2}$ Image Processing Lab, Department of Electrical Engineering, \\ Maharana Pratap A\&T University, Udaipur Rajasthan, India; ${ }^{3}$ Biological Engineering, Utah State University, Logan, USA. \\ Email: rs2010@,columbia.edu; soonjo.kwon@usu.edu
}

Received 23 August 2008; revised 20 July 2009; accepted 25 July 2009.

\section{ABSTRACT}

Background: In the last decade, sodium magnetic resonance imaging was investigated for its potential as a functional cardiac imaging tool for ischemia. Later interest was developed in contrast enhancement for intracellular sodium. Little success was reported to suppress extracellular sodium resulting in the intracellular sodium MRI image acquisition using quantum filters or sodium transition states as contrast properties. Now its clinical application is expanding as a new challenge in brain and other cancer tumors. Contrast enhancement: We highlight the physical principles of sodium MRI in three different pulse sequences using filters (single quantum, multiple quantum, and triple quantum) meant for sodium contrast enhancement. The optimization of scan parameters, i.e. times of echo delay (TE), inversion recovery (TI) periods, and utility of Dysprosium (DyPPP) shift contrast agents, enhances contrast in sodium MRI images. Inversion recovery pulse sequence without any shift reagent measures the intracellular sodium concentration to evaluate ischemia, apoptosis and membrane integrity. Membrane integrity loss, apoptosis and malignancy are results of growth factor loss and poor epithelial capability related with MRI visible intracellular sodium concentration. Applications and limitations: The sodium MR imaging technical advances reduced scan time to distinguish intracellular and extracellular sodium signals in malignant tumors by use of quantum filter techniques to generate $3 \mathrm{D}$ sodium images without shift regents. We observed the association of malignancy with increased TSC, and reduced apoptosis and epithelial growth factor in breast cancer cells. The validity is still in question. Conclusion: Different modified sodium MRI pulse sequences are research tools of sodium contrast enhancement in brain, cardiac and tumor imaging. The optimized MRI scan parameters in quantum filter techniques generate contrast in intracellular sodium MR images without using invasive contrast shift agents. Still, validity and clinical utility are in question.

Keywords: Sodium MRI; Double Quantum; Inversion Recovery; Contrast Enhancement; Cancer

\section{BACKGROUND}

Sodium-23 (Na-23) nuclei are in abundance in the body but exhibit poor magnetic resonance sensitivity and serve as sodium MRI clinical imaging modalities. The sodium Na-23 nucleus is detectable by MRI due to its $3 / 2$ spin existing as $-3 / 2,-1 / 2,0,1 / 2,3 / 2$ (5 states). Physical nature of sodium-23 with spin $=3 / 2$ is suitable to have a nonvanishing magnetic moment and Larmor frequency $=11.26 \mathrm{MHz}$ and exhibits four transition states with five different energy levels in the static magnetic field. MR detection sensitivity is very low $(9.3 \%)$ at in vivo concentration (approximately $0.05 \%$ ).

Sodium is abundant $(100 \%)$ in living body tissue in $3 / 2$ spin state. The sodium ion is the predominent cation in the extracellular fluid $(139 \mathrm{mmol} / \mathrm{L})$, while its intracellular concentration is very low (in the order of 8-10 $\mathrm{mmol} / \mathrm{L}$ ), mainly bound with glycosides and proteins. The role of increased intracellular sodium due to concerted efflux through $\mathrm{Na}^{+} / \mathrm{K}^{+}$ATP-ase intrinsic action, angiogenesis, and cell proliferation was described in ischemia, hypoxia, arrhythmia, myocardial edema, and tumors [1]. The sodium concentration is sensitive to disease as an indicator of cellular and metabolic integrity. $\mathrm{Na}-23$ concentration in tissues is present in the order of tens of millimoles. The low sodium concentration results in low signal-to-noise ratio of ${ }^{23} \mathrm{Na} \mathrm{MR}$ imaging in long imaging times and/or poor spatial resolution. Therefore, it needs the use of shift reagents or pulse sequences with 
filters such as single-, double-, triple-, and multiple quantum (SQ, DQ, TQ and MQ) filters to enhance the sodium contrast [2].

Evidence of sodium transfer rates across the bloodbrain barrier dates back to its use in MRI [3] followed by the use of spin echo pulse sequence for experimental sodium imaging with first surface coil [4]. Soon after it, in vivo clinical sodium imaging at clinical $1.5 \mathrm{~T}$ scanners was first described using multislice spin echo pulse sequences [5]. Later developments were made at high magnetic field MRI scanners using ultrashort echo time sequences and hardware improvements that permitted better spatial resolution with shorter imaging times and better quantitative measurements of tissue sodium concentration without use of shift reagents [6].

This paper describes the phases and roles of quantum filters in pulse sequence design to generate intracellular/extracellular sodium contrast, high signal-to-noise ratio, and high resolution in magnetic resonance sodium images by triple-quantum filter and double inversion recovery pulse sequence without using paramagnetic shift reagents. Triple quantum filter was evaluated as a superior method for high signal-to-noise ratio but needs a long acquisition time [7]. Other available multiplequantum, single-, and double quantum filter tomographic magnetic resonance pulse sequences generate intracellular sodium images with high signal-to-noise ratio but need paramagnetic shift reagents as contrast agents.

\subsection{Nature of Sodium MR Signal}

At high field magnetic resonance application, the applied phase-cycled radio frequency pulses at set time intervals cause an alignment of nuclei populations within the sample, resulting in a measurable signal in the transverse plane. Optimized times of echo delay (TE) in single-, double-, triple quantum filters; optimized times of inversion times (TI) for inversion recovery pulse sequence; and application of a multiple quantum filter after injection of paramagnetic shift contrast reagents detected the distinct intracellular sodium signal to obtain a sodium weighted image. As a result, two sodium MRI signals are achieved with distinct MRI signal intensities to measure sodium intracellular and extracellular concentrations in the tissue. The MR signal intensity is related with tissue sodium content as follows: The MR signal intensity of $\mathrm{Na}-23$ is:

$$
\text { MR sensitivity }=\% \mathrm{Na}-23 / \mathrm{H}-1 . \mathrm{C}(\mathrm{Na}-23) . \text { A. } \gamma . \mathrm{S}^{2}+\mathrm{S} \text {, }
$$

where $\% \mathrm{Na}-23 / \mathrm{H}-1$ is sodium abundance, $\mathrm{C}(\mathrm{Na}-23)$ is tissue concentration, $\mathrm{A}$ is MR scanner intrinsic parameter, $\gamma$ is Gyromagnetic Ratio and $\mathrm{S}$ is nuclear spin.

Due to the Gyromagnetic Ratio of Na-23 $=11.25$ $\mathrm{MHz} / \mathrm{T}$ relative MR sensitivity is lesser. Moreover, sensitivity is further decreased due to less sodium content in the tissue: about $1 / 1000$ th MR sensitivity of proton, so it needs use of high magnetic field imaging scanner and data acquisition techniques with contrast enhancement mechanisms, such as MQ, SQ, DQ and TQ. Sodium concentration is higher in blood than that in myocardium and other tissues. This high sodium density in blood contributes to tissue contrast in sodium MR imaging. However, a very small amount of $\mathrm{Na}-23$ is MR visible in free ionic extracellular form due to poor sensitivity. Other intracellular forms of invisible sodium are mainly bound to proteins and phospholipids in the tissue, so intracellular sodium MRI visualization needs quantum filters.

\subsection{Quadruple Interactions and Sodium MR Signal}

Sodium signal is conventionally represented as quadruple interactions between Na-23 and its molecular environment. In sodium nuclei, magnetic resonance excitation and subsequent transition of electrons to different energy levels of nuclear spins produce single-, double-, triple-, and multiple quantum sodium transition MR signals as said earlier. These different quantum transitions can be filtered by use of specific SQ, DQ, TQ and MQ pulse sequence techniques by use of selective phases in pulse sequence design as described later. These techniques also produce spectral patterns reflecting the percentage of invisible intracellular sodium. These sodium spectra are iterated superimposed positions of different spectra and represent simulated signals from intracellular sodium in the cytoplasm including interstitium, mitochondria, intravascular space, and so on. However, this invisible "intracellular" $\mathrm{Na}-23$ results from the presence of a broad frequency peak (T2, very short component). It masks the corresponding frequency peak. Another contributory factor to intracellular Na-23 MR invisibility is long correlation times for short and long T2 components. For the heart, the correlation time is in the range of $10^{-12}$ to $10^{-9}$ seconds. As a result, intracellular sodium is invisible. The double quantum pulse technique (instead of single quantum pulse technique) selects only double quantum transitions of sodium. These double quantum transitions allow sodium to be more ordered with long correlation time. These pulse sequence techniques visualize the intracellular sodium.

\subsection{Nature of Sodium Nuclei and MRI Properties}

The Larmor frequencies of sodium in extracellular and intracellular sodium populations are the same because they have the same resonant frequencies. Therefore, three different approaches are used in distinguishing these two types of sodium nuclei. These include different relaxation times between intracellular and extracellular sodium, the use of paramagnetic shift reagents, and the use of special multi-quantum pulse sequences. 


\subsection{Sodium Relaxation Times}

Based on the observation that tissue transverse relaxation time for the intracellular space is smaller than that of extracellular space, intracellular space can be imaged by shortening the spin-echo time. Intracellular sodium signal intensity proportionally increases due to intracellular space. Sodium transverse relaxation time (T2) is biexponential in nature, while sodium is bound with negatively charged macromolecules - mainly proteins or metabolites. Perhaps the longitudinal relaxation time (T1) also behaves as biexponential.

\subsubsection{Paramagnetic Shift Reagents}

Paramagnetic shift reagents are chelates of paramagnetic lanthanide ions with anionic complexes. These substances don't cross the intact cell membranes. These reagents interact with extracellular sodium and introduce a frequency shift in the resonance frequency of extracellular sodium. This shift allows the advantage of producing two separate spectral peaks arising from intracellular and extracellular sodium. Common lanthanide ions used as paramagnetic shift reagents are $\mathrm{Dy}^{3+}$ (Dysprosium), $\mathrm{Tm}^{3+}$ (Thulium), $\mathrm{Gd}^{3+}$ (Gadolinium), $\mathrm{PPP}^{5-}$ (Tripolyphosphate), and TTHA ${ }^{6-}$ (Tetraethylene Triaminehexa acetic acid). These reagents bind with intracellular calcium and cause physiological disturbances and, in some cases, toxicity, so their use is limited. To overcome such problems, several approaches were recently developed based on the modification of pulse sequences and hardware improvements as described in the following section.

\subsection{Quantum Filters}

These are MR pulse sequences that allow direct observation of multiple quantum transitions. These filters usually are constructed by phase cycling manipulations. The general pulse sequence scheme is $90^{\circ}-\mathrm{t} / 2-180^{\circ}-\mathrm{t} / 2-90^{\circ}$ $-\pi-90^{\circ}$-acquire, where $t$ is creation time and $\pi$ is evolution time by use of pulsed field gradients. Characteristically, these filters are sensitive to variations in phase cycles up to $5^{\circ}$ and magnetic field inhomogeneity. The filter can be single-, double-, triple-, or multiple quantum and allow single, double-, triple-, and multiple quantum transitions. In the following sections, we introduce readers to our strategy of quantum filters used in sodium MRI pulse sequence design.

\subsubsection{Single Quantum Pulse Sequence}

Due to its multiple spin state transitions, an alternative MR single quantum approach is used to measure intracellular $\mathrm{Na}$ content based on the interaction of $\mathrm{Na}$ polyanions and their resultant effects on nuclear spin transitions. Spin 3/2 nuclei (such as Na-23) have a nonvanishing quadrupole moment, allowing interaction with electrostatic field gradients. The pulse sequence is a spin echo sequence.

\subsection{Multiple Quantum Pulse Sequence for Origin of Double- and Triple Quantum}

For intracellular sodium, using only multiple-quantum (MQ) NMR requires paramagnetic shift reagents (SRs) that have distinct disadvantages including: toxicity, possible drug interaction, expanded space, and impermeability to the blood brain barrier. It is known that the correlation time, $t_{c}$ of the time variations of the electrostatic field gradients in spin 3/2 nuclei satisfies the relation if $\mathrm{wLt}_{\mathrm{c}} \gg 1$, where $\mathrm{wL}$ is the Larmor frequency. These nuclei can display biexponential relaxation and MQ spin transitions do occur in the nuclei and these transitions are detected by specific pulse sequences called multiple- quantum filters.

A phase-cycled RF pulse sequence applied over a slice-select axis selects both echo and anti-echo signals of spin undergoing multiple-quantum spin transitions at resonating Larmor frequency. The RF pulse has both a flip angle and a phase angle. The phase angles are cycled using multiple-quantum transition filter and simultaneously produce an output signal proportional to the sum of echo and anti-echo signals of MQ coherence. It includes the evolution period with a flip angle of $180^{\circ}$ to refocus the RF pulse to avoid inhomogeneity-induced amplitude deterioration. This output signal is measured by induction current in the RF coil during the realignment process and is used in tomographic MQ transition ${ }^{23} \mathrm{Na}$ MRI images.

The pulse sequence comprises: a preparation period of length $t_{p}$ extending from time $t_{0}$, an evolution period of length $t_{e}$ and a detection period of length $t_{d}$. The RF pulse sequence manipulates the selected nuclei to exhibit them with single quantum coherence. During the evolution period, the pulse sequence implements a MQ filter which isolates the coherence (e.g. double- or triple quantum) and simultaneously selects both echo and antiecho signals corresponding to the coherence by; a) an evolution period first flip angle RF pulse timewise centered at $t_{p}$ which converts the selected nuclei from single-quantum coherence to the selected multiple- quantum coherence; b) an evolution period of second $90^{\circ}$ flip angle RF timewise centered at $t_{p}+t_{e}$, converts the selected nuclei from multi-quantum to single-quantum coherence. Therefore, during the detection period, selected nuclei exhibit single quantum coherence to generate a single quantum signal.

The resultant collective phase angle of evolution period RF pulse $=\Phi_{\mathrm{E}}=\Phi_{3}-2 \Phi_{4}+\Phi_{5}$, wheree $\Phi_{\mathrm{E}}$ is collective radio frequency phase during evolution time, and $\Phi_{4} \&$ $\Phi_{5}$ are phases of $90^{\circ} \mathrm{RF}$ pulses. A further preparation period of $90^{\circ}$ flip angle radio frequency slice-selecting pulse, a preparation $180^{\circ}$ flip angle refocusing pulse, and a detection period $180^{\circ}$ flip angle radio frequency are applied - centered at $t_{0}, 1 / 2 t_{\mathrm{p}}$ and $t_{\mathrm{p}}+t_{\mathrm{e}}+1 / 2 \mathrm{t}_{\mathrm{d}}$, respectively. The preparatory $90^{\circ}$ flip angle RF pulse selects a 
slab of sample perpendicular to a slice-selection axis as shown in Figure 1. The preparatory $90^{\circ}$ flip angle slice-selection RF pulse has phase angle $\Phi_{1}$, the preparatory $180^{\circ} \mathrm{Rf}$ refocusing pulse has $\Phi_{2}$, and detection period $180^{\circ}$ flip angle RF pulse has phase angle $\Phi_{\mathrm{P}}$ of the RF pulses during preparation time (evolution period $90^{\circ}$ flip angle RF phase $\Phi_{3}$ ) as described earlier(8). The phase angle is $\Phi_{\mathrm{P}=} \Phi_{1}-2 \Phi_{2}+\Phi_{3}$ and the output signal will be $\Phi=\Phi_{\mathrm{P}-\mathrm{m}} \Phi_{\mathrm{E}^{+}} \Phi_{5+2} \Phi_{6+} \Phi_{\mathrm{R}}$, where $\mathrm{m}$ is the integer of magnetization, $\Phi$ is the phase of detected signal, and $\Phi_{R}$ is the phase of receiver.

For double quantum filtered images, $\mathrm{m}=+2$, and for triple quantum filtered images, $m=+3$. The phases will be $\Phi 1=(\mathrm{n} / 2) \pi, \quad \Phi_{2}=[\mathrm{n} / 2+\operatorname{int}(\mathrm{n} / 4)] \quad \pi, \quad \Phi_{3}=(\mathrm{n} / 2) \pi$, $\Phi_{4}=\Phi_{5}=\Phi_{6}=0$ and $\Phi_{\mathrm{R}}=\mathrm{n} \pi$ for double quantum filter; and phases will be $\Phi 1=(\mathrm{n} / 3) \pi, \quad \Phi_{2}=[\mathrm{n} / 3+\operatorname{int}(\mathrm{n} / 6)] \pi$, $\Phi_{3}=(\mathrm{n} / 3+1 / 2) \pi, \quad \Phi_{4}=0, \quad \Phi_{5}=(1 / 6) \quad \pi, \quad \Phi_{6}=0 \quad$ and $\quad \Phi \mathrm{R}$ $=[n+\operatorname{int}(n / 6)] \pi$ for triple quantum filter, where $n / 6$ is integer part of the variable $n / 6$. The variable $n$ is positive integer of $0 \ldots \mathrm{N}-1 . \mathrm{N}$ is a multiple of 8 and 12 for double- and triple quantum filters respectively.

The magnetic gradient pulse sequence is also applied along with the RF pulse sequence for positional information in the data. A slice-select gradient pulse, first and second phase encoding gradient pulse, and read-out gradient pulse are applied along read-axis after detection period $180^{\circ} \mathrm{RF}$ refocusing pulse during output signal observed as shown in Figure 1. The read-out pulse area before time $t_{P}+t_{e}+t_{d}$ is equal to the area of read-out gradient pulse. These gradient pulses exhibit simultaneous synchrony with RF pulses.

\subsubsection{Inversion Recovery Pulse Sequence}

The inversion recovery technique can suppress the extracellular sodium nuclei with specific ranges of the lon-

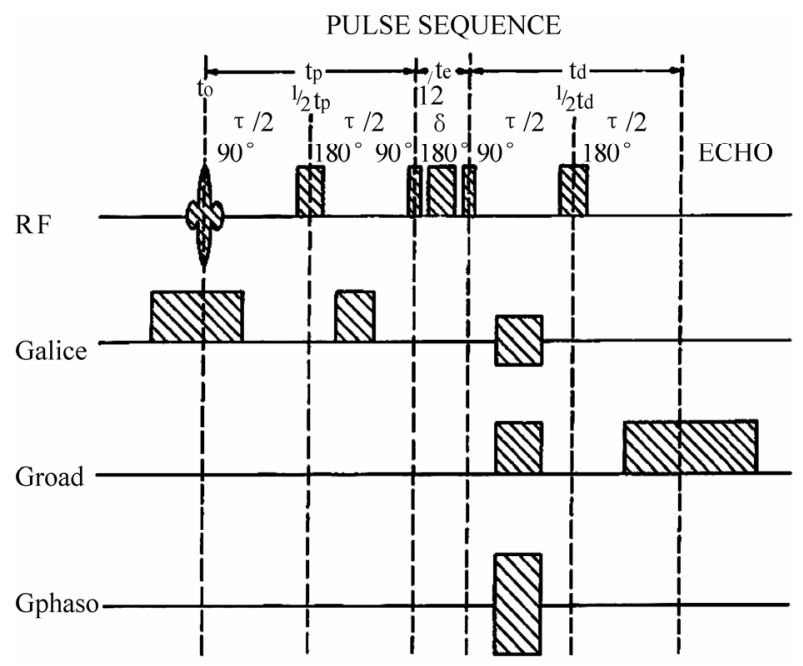

Figure 1. The figure illustrates a series of radiofrequency pulses and a synchronized series of magnetic gradient pulses for phase cycled evolution period $180^{\circ}$ flip angle refocusing pulse.

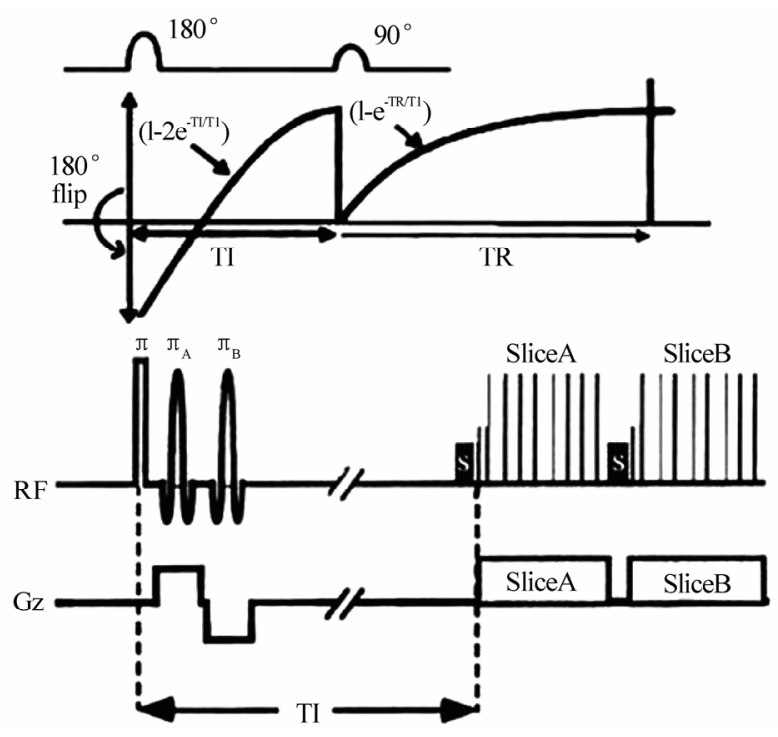

Figure 2. A scheme of inversion recovery pulse sequence is presented. On top, one cycle of IR with two recovery curves is shown for a single slice. First, $180^{\circ}$ pulse is flipping the magnetization $\mathrm{Mz}$ for first recovery followed by $90^{\circ}$ pulse for FID and second recovery within TR period. At bottom, the application of two inversion pulses $\pi \mathrm{A}$ and $\pi \mathrm{B}$ is shown for slices A and B with simultaneous application of RF and gradient pulses.

gitudinal relaxation time, T1 using fast spin-echo pulse sequence. The sequence has two sets of slice selective inversion pulse trains (long recovery period $\mathrm{TI}_{1}$ for extracellular sodium and short recovery period $\mathrm{TI}_{2}$ for intracellular sodium nuclei). The first inversion pulse is played during a long recovery period $\mathrm{TI}_{1}$, during which extracellular sodium magnetization recovers just past its null point while longitudinal magnetizations of intracellular sodium nuclei are almost fully restored. The second set of inversion pulse is played during short recovery period $\mathrm{TI}_{2}$, when intracellular $\mathrm{Na}$ nuclei magnetizations reach their null points following $\mathrm{TI}_{2}$ interval and become $180^{\circ}$ out-of-phase. After inversion pulses, a fast spinecho acquisition sequence is applied to achieve magnetization in $\mathrm{z}$-direction $(\mathrm{Mz})$ as following:

$$
M z=M_{0}\left[\left(1-2 e^{-T I e / T l e}+e^{-T R / T l e}\right)\left(1-2 e^{-T I i / T l i}\right)\right.
$$

where 'e' represents extracellular sodium nuclei and ' $i$ ' represents intracellular sodium nuclei. $\mathrm{M}_{0}$ is initial longitudinal magnetization, $\mathrm{TI}_{1} \& \mathrm{TI}_{2}$ are long and short inversion times, and $\mathrm{T}_{1 \mathrm{e}} \& \mathrm{~T}_{1 \mathrm{i}}$ are longitudinal relaxation times of extracellular and intracellular sodium nuclei, respectively.

The relaxation time for intracellular $\mathrm{Na}$ nuclei varies from 5-10 milliseconds (for Na bound to large molecules or dispersed in highly order environment) to 51 milliseconds (for extracellular sodium nuclei in free solution). Based on $3 \mathrm{D}$ projection reconstruction imaging pulse sequence, echo time TE as short as $0.1 \mathrm{~ms}$ can be 
achieved. This captures the more rapid decay of short $\mathrm{T}_{2}$ sodium components in the NMR signal so as to further improve the signal to noise (SNR).

As shown in Figure 2, radio frequency pulse at $180^{\circ}$ is followed by a $90^{\circ}$ pulse for slice excitation utilizing echo-train length with any option of selective or nonselective, single or composite, sinc, sech or tyco pulse as needed. So, inversion pulses are played out during inversion $\mathrm{TI}_{1}$ and $\mathrm{TI}_{2}$ periods for both types of sodium nuclei. Data acquired includes projection reconstruction with slice selection or without slice selection using spin-echo or gradient echo. Thus, inversion/acquisition periods are played out for several slices per repetition of fast spin echo (FSE) sequence exhibiting two distinct null points for both sodium nuclei populations.

\section{MRI PHYSICS OF CONTRAST ENHANCEMENT BY INVERSION RECOVERY AND TWO NULL POINTS}

After first $180^{\circ} \mathrm{RF}$ pulse flips the magnetization from $\mathrm{M}_{-\mathrm{z}} \rightarrow \mathrm{M}_{\mathrm{z}}$, the two different inversion times $\left(\mathrm{TI}_{1}\right.$ and $\left.\mathrm{TI}_{2}\right)$ for two sodium populations exhibit two null points and growth of longitudinal magnetizations. In this sequence, soon after TI, a $90^{\circ} \mathrm{RF}$ pulse is applied to flip the longitudinal magnetization into the $x-y$ plane for the $T_{1}$ growth curve within repetition time TR. At this point, frequency induction decay (FID) is measured when the longitudinal magnetization is flipped into the $\mathrm{x}-\mathrm{y}$ plane. Thereafter $180^{\circ} \mathrm{RF}$ pulses are repeatedly applied. The magnetization signal ' $\mathrm{S}$ ' may be measured as: [S] $\alpha$ $\mathrm{M}_{0}\left(1-2 \mathrm{e}^{-\mathrm{TL} / \mathrm{T} 1}\right)\left(1-\mathrm{e}^{\mathrm{TR} / \mathrm{T} 1}\right)$, as $\mathrm{T}_{1}$ recovery curves for both $180^{\circ}$ and $90^{\circ}$ degree pulses applied.

The intracellular and extracellular populations of nuclei have different longitudinal relaxation times, so they generate intracellular/extracellular sodium contrast. To set the IR pulse sequence to suppress the contribution of sodium nuclei with specific ranges of $T_{1}$, the inversion time $\mathrm{TI}_{1}$ or $\mathrm{TI}_{2}$ is set as $(\ln 2)\left(\mathrm{T}_{\text {lex }}\right)$, where $\mathrm{T}_{\text {lex }}$ is the composite longitudinal relaxation time. This inversion time then determines the time between the $180^{\circ}$ and $90^{\circ}$ pulses in the IR pulse sequence.

\subsection{Projection Reconstruction Methods}

First, projection reconstruction was proposed based upon Fourier transformation of the FID (free induction decay) signals [9].

Now, these FID signals are obtained by rotating a magnetic field gradient with back-projection to determine the spin-density function or enhanced contrast. So far, this technique has been used only for animal hearts. These signals can be gated to heart rate to reduce the motion artifact. Signals are reconstructed from 12 projections. Each projection is obtained from an average of 320 FID signals. For image matrix $64 \times 64$ total data acquisition, time is approximately 15 minutes.

\subsection{Three-Dimensional Fourier Techniques}

In this technique, phase-encoding is made in two orthogonal directions, and a fixed gradient is applied in a third direction of read-out gradient [10]. The pulse- encoding steps are applied, typically 40 steps along each phase-encoding direction. As a result two echo signals with different spin-echo times are generated and added in a coherent manner to enhance contrast. This phenomenon also improves the signal-to-noise ratio. This technique is used for the human brain. In cardiac sodium imaging the utility of this technique is limited to animal heart experiments. Typically short TR $=100 \mathrm{~ms}$ is used to get data in 3 hours, including different spin echoes summation. The application of greater sodium signal in the left anterior descending artery was observed in occlusion, followed by re-perfusion of circumflex in the coronary artery after ischemia.

\subsection{Hybrid Spin Echo Techniques}

The spin-spin T2 relaxation time of intracellular sodium is shorter than that of the extracellular sodium compartment, so it is difficult to observe it to create contrast, and it needs a hybrid spin-echo pulse sequence. In the hybrid approach, both projection-reconstruction and Fourier encoding schemes are used at the same time. Fourier analysis does slice selection in one direction while another projection-reconstruction is performed in two other orthogonal directions. This technique is good for clinical MR scanners with gradient refocused sequence with modified head coil to generate echo time as short as 2.8 msec [11].

\subsection{Surface Coil Techniques and Coil Sensitivity}

Sodium imaging at short $T_{2}$ relaxation times can be performed by use of a customized radio-frequency coil focused on the region-of-interest to improve the signal-tonoise ratio. The pulse sequence is applied with optimizing the minimum saturation time of preamplifier and duration of 90-degree RF pulse. This effect minimizes the magnetic homogeneity influence over the free induction image and the short $T_{2}$ can be imaged. This technique is relatively better than others for human cardiac sodium imaging on clinical scanners. First, using different transmitter gains optimizes the gain for $90^{\circ}$ pulse, and different transmitter gains at increased and decreased gain values yields ${ }^{23} \mathrm{Na}$ images at different flip angles of $45^{\circ}, 90^{\circ}$, and $135^{\circ}$ with matrix sizes $32 \times$ $32 \times 32$ points. Nonlinear least squares fit of the pixels intensities in all three images determines the B1 field strength (for $5 \%$ maximum intensity on image) as a function of the related [Na]i image intensities in response to the local RF coils for receive and transmit sensitivities [13]. 
The image signal intensity depends on coil transmit and coil receive sensitivity as:

$$
I_{k}=R_{k} M_{0 k}\left|\sin \left(\Phi_{k}\right)\right|
$$

where $I_{k}$ is the signal intensity of the $k_{t h}$ pixel, $R_{k}$ is a sensitivity at the coil center, and $\mathrm{M}_{0 \mathrm{k}}$ is the equilibrium magnetization. The flip angle $\Phi_{\mathrm{k}}$ is a function of the transmitter gain setting (TG) as:

$$
\Phi_{k}=\left(C R_{k)}\right) 10^{T G / 20}
$$

$\left(\mathrm{C} \cdot \mathrm{R}_{\mathrm{k}}\right)$ and $\mathrm{M}_{0 \mathrm{k}}$ were each fitted with a single parameter for each pixel. The constant ' $\mathrm{C}$ ' is independent of spatial position and receives sensitivity. It is also directly proportional to the transmit field distribution for the coil [13]. After normalization, the product $\left(C \cdot R_{k}\right)$ yields the relative sensitivity $R_{k}$ for the $k_{t h}$ pixel. The $B_{1}$ (expressed as $\mathrm{B}_{1} / 2$ ) depends on reference transmit power, $\mathrm{TG}_{\mathrm{ref}}$ :

$$
\frac{\gamma B_{1 k}}{2 \pi}=\left(C R_{k}\right) 10^{T G_{r e f} / 20} /\left(t_{p}(2 \pi)\right)
$$

where $t_{p}$ is the pulse duration.

Local field $\mathrm{B} 1$ and longitudinal relaxation time $\mathrm{T} 1$ determine the correction factors for saturation for each pixel $\mathrm{k}, \mathrm{SF}_{\mathrm{k}}$ as follows:

$$
S F_{k}=\frac{\left[-e^{-T R / T 1}\right] \sin \left(t_{p} \frac{\gamma B_{1 k}}{2 \pi}\right)}{\left[1-e^{-T R / T 1} \cos \left(t_{p} \frac{\gamma B_{1 k}}{2 \pi}\right)\right]}
$$

where $B_{1 k}$ is the $B_{1}$ field in the respective ROI and is the gyromagnetic ratio for $[\mathrm{Na}]_{i}$ using the pulse time $t_{p}=0.4$ msec, and the TR=120 msec [13].

\subsection{Rotating Frame Techniques}

Conventionally, sodium imaging can be done using the rotating frame technique based on superimposing a magnetic field gradient on the excitation field $B_{1}$. As a result, during this excitation period, spatial localization and spatial encoding may be performed very fast to generate sodium contrast. In this process, loss of MR signal intensities is also minimized. In an isolated perfused rabbit heart, sodium-rotating frame imaging experiment was completed in 12 msecs in a $128 \times 128$-image matrix with a clinical $1.5 \mathrm{~T}$ scanner [12].

\subsubsection{Use of Contrast Agents}

Different shift agents such as dysprosium or Tm (DOTP) contrast agents increase the sensitivity and specificity of sodium MRI by shifting intracellular sodium magnetization away from extracellular sodium magnetization (exhibited well as shifted peaks). Dextran-magnetite increases the contrast between tumor and surrounding soft tissue sodium images. Normal reticulo-endothelial cells take up a greater amount of magnetic particles than tumor cells do, which supports the potential for tumor so- dium imaging by contrast agents. However, a major role of sodium imaging seems to distinguish myocardial ischemia.

Techniques used for sodium in vivo MRI: Several approaches have been used. Despite little success, these techniques are gaining interest in physiological MR imaging using intracellular sodium. Some landmark techniques have been described for image generation.

\subsubsection{Spectrally Weighted Twisted Projection Imaging}

The 3D sodium MRI imaging can be made faster by reducing the $T_{2}$ signal attenuation effects. This is implemented by 3D twisted projection imaging. At high spatial frequencies, the sample density is reduced, and as a result the reduced readout time is achieved. This leads to decreased $\mathrm{T}_{2}$ signal attenuation, which translates into improved signal-to-noise ratio (SNR) without a loss of resolution [14].

\subsubsection{Biexponential Relaxation Effect and Sodium Concentration Mapping}

Dual-frequency RF coils with identical B1 field distributions at the two different observation frequencies provide sodium and proton mapping. These optimize both dual frequencies in dual quadrature RF coils. Sodium and proton channels are decoupled to make dual-quadrature birdcage configurations. The fourth harmonic of sodium frequency happens to be very close to proton frequency. This is suitable for echo-planar imaging and metabolite quantification [15].

\subsubsection{3-D Triple Quantum-Filtered Twisted Projection Na-23 Imaging}

A new approach was developed based on a three pulse, six-step, coherence transfer filter (with fast twisted projection imaging sequence) to generate spatial maps of the TQ signal. In principle, three pulse coherence filter leads to TQ sodium images. In these images, the image intensity depends on the spatial variation of the flip angle. This image intensity is lesser than the flip angle chosen in the four pulse TQ filter. This technique allows the generation of RF inhomogeneity corrected TQ sodium images after TQ signal variation. The pulse sequence of TQ filtering is based on spherical tensor operators or a density matrix. It consists of three non-selective RF pulses, and sometimes a fourth RF pulse between the three first and the second pulses, to refocus the main magnetic field inhomogeneities [16]. In this pulse sequence, RF induces different flip angles as $\theta_{30}, 2 \theta_{30}$, $\theta_{150}, \theta_{0} \ldots$ flip angles where the phase stepped up through the values $30^{\circ}, 90^{\circ}, 150^{\circ}$, and so on. The second pulse is intended for the B inhomogeneities refocusing and is absent in the case referred to as "three pulse". The preparation time is the period between two phases and evolution time is the period between $\theta_{150}$ and $\theta_{0}$ 


\subsection{Simultaneous Quadruple and Double Quantum Na-23 MR Imaging}

$\mathrm{Na}-23$ is a quadruple nucleus with biexponential relaxation or non-vanishing quadruple coupling. Both properties contribute to generate $\mathrm{Na}-23$ multiple-quantum (MQ) coherence [17]. The Multiple Quantum Filter pulse sequence can be represented as Eq.6:

$$
\left(\theta_{1}, \varphi_{1}\right)-\mathrm{t}_{\mathrm{P}} / 2-\left(\theta_{2}, \varphi_{2}\right)-\mathrm{t}_{\mathrm{P}} / 2\left(\theta_{3}, \varphi_{3}\right)-\mathrm{t}_{\mathrm{E}}-\left(\theta_{4}, \varphi_{4}\right)-\operatorname{Acq}(\mathrm{T}, \varphi \mathrm{R}),(6)
$$

where refocused preparation time, $\varphi_{P}$ and non-refocused evolution time, $\varphi_{\mathrm{E}}$ may be represented as Eq.7:

$$
\varphi_{P}=\varphi_{1}-2 \varphi_{2}+\varphi_{3} ; \varphi_{E}=-\varphi_{3}+\varphi_{4}
$$

where $\varphi_{1-4}$ denote the phase of each RF pulse with flip angle $\theta_{1-4}, \varphi R$ is receiver phase, and $T$ is acquisition time, each $\theta_{1}=\pi / 2$ and $\theta_{2}=\pi$.

This sequence is good for detecting well-defined and ordered structures in biological tissues. Both quadruple-order and double-quantum signals can be generated simultaneously by the same RF sequence without any loss in signal amplitude. In addition, the acquired MQ signals can be readily decomposed into their second and third - rank components, i.e. into quadruple order and biexponential relaxation components. This allows reduction of the scan time to acquire MQ signals of different coherence orders and ranks, which should prove useful for in vivo studies.

\subsection{Double Spin-Echo Imaging and Transverse Relaxation Time}

This approach of imaging is good for myocardial water content analysis based upon the calculation of transverse relaxation time from dual-quantum spin-echo MRI [18]. Right and left ventricles can be assessed for quantification of myocardial edema. The transverse relaxation times $\left(\mathrm{T}_{2}\right)$ of ventricles can be calculated from the signal intensities within multiple regions of interest over myocardium as shown in Eq.8:

$$
\mathrm{T}_{2}=\left[\left(\mathrm{TE}_{2}-\mathrm{TE}_{1}\right) / \ln \left(\mathrm{I}_{1} / \mathrm{I}_{2}\right)\right],
$$

where $\mathrm{TE}_{1}$ is first echo time, $\mathrm{TE}_{2}$ is second echo time, and $I_{1} \& I_{2}$ are image amplitudes of first \& second spin echoes.

\subsection{Double-Quantum-Filtered Na-23 MR Spectroscopy}

The extracellular EC-Na and intracellular IC-Na contents are better visualized by multiple-quantum-filter spectra acquired in the absence of chemical shift or relaxation reagents. The intracellular IC-Na sensitivity can be enhanced by double-quantum-filter (DQF) Na-23 NMR to measure the detection of $\mathrm{Na}^{+}$motion anisotropy with the presence of residual quadruple splitting (19). However, the application of this technique is limited to the experimental heart studies using the pulse sequence: $90^{\circ}-\tau / 2-180^{\circ}-\tau / 2-\theta-\mathrm{t}_{1}-\theta-\mathrm{t}_{(\mathrm{Acq})}$, where $\tau$ denotes the creation time and $\mathrm{t} 1$ is evolution time, $\theta$ is RF flip angle.

The magnetization $\mathrm{M}(\tau, \mathrm{t}, \theta)$ for the MQF Na-23 NMR signal, when the quadruple splitting factor $(\omega Q)$ is zero, is given by Eq.9:

$$
\begin{aligned}
\mathrm{M}(\tau, \mathrm{t}, \theta)= & \alpha \mathrm{M}_{0}\left[\exp \left(-\tau / \mathrm{T}_{2 \mathrm{~s}}\right)-\exp \left(-\tau / \mathrm{T}_{2 \mathrm{f}}\right)\right] \\
& \mathrm{x}\left[\exp \left(-\mathrm{t} / \mathrm{T}^{*}{ }_{2 \mathrm{~s}}\right)-\exp \left(-\mathrm{t} / \mathrm{T}^{*}{ }_{2 \mathrm{f}}\right)\right] \\
& \mathrm{x} \sin ^{2} \theta\left(1-3 \cos ^{2} \theta\right)
\end{aligned}
$$

where $\alpha$ is a factor which depends on the degree of quantum coherence, $\mathrm{M}_{0}$ is equilibrium magnetization, $\mathrm{T}_{2 \mathrm{f}} \& \mathrm{~T}_{2 \mathrm{~s}}$ are fast $\&$ slow transverse relaxation times, and $\mathrm{T}^{*}{ }_{2 \mathrm{f}} \& \mathrm{~T}^{*}{ }_{2 \mathrm{~s}}$ are the corresponding inhomogeneitybroadened times. An important implication for this technique is the possibility of measuring intracellular sodium concentration in animal hearts with the DQF spectrum in the absence of shift or relaxation reagents. Significant attenuation of the DQF spectrum derived from extracellular $\mathrm{Na}^{+}$allows to differentiate the NMR spectrum from intracellular $\mathrm{Na}^{+}$.

\subsection{Double Quantum Filtering and Spin-Quantum Coherence}

Conventionally, the double-quantum filtering method for sodium imaging is completed in a four-step phase-cycling scheme. In this process, the radio-frequency pulses generate single-quantum coherence. Its phase appears similar to the phase of a double-quantum coherence signal in the pulse sequence. Consequently, the intersequence stimulated echo passes through the doublequantum filtration in the phase cycling scheme and gradient pulses in the DQ pulse sequence, so the single-quantum coherence is an unwanted component in this pulse sequence. It is eliminated by the use of spoiling RF pulses followed by dephasing gradient pulses incorporated into the DQ filtering pulse sequence. These spoiling pulses disperse the pulse transition and eliminate the magnetization components of the intersequence stimulated echo in the DQ pulse sequence [20]. Another good way to eliminate single quantum coherence is to increase the repitition time TR. Moreover, a low signal level of DQ coherence also demands many signal averaging at short TR to keep short scan time and not a longer TR.

\subsection{Chemical Shift Selective Acquisition of Multiple Quantum-Filtered Na-23}

In cardiac imaging, MQ signals over a wide off-resonance bandwidth is a problem which causes interference between echo and anti-echo. It is due to a resonance offset insensitive to the flip angle of the creation RF pulse; usually the second $\pi / 2$ pulse. Off-resonance effects are applied to eliminate the MQ signal in the presence of a chemical shift. It suppresses the MQ signal over a wide range of off-resonance bandwidth [21]. Existing tech- 
niques for chemical-shift-selective acquisition of SQ signals can be combined with MQ off-resonance effects to enhance the selectivity of chemical shift.

\subsection{Double Quantum Filtering and Elimination of Intersequence Stimulated Echo}

In cardiac sodium imaging the 'intersequence stimulated echo' is an unwanted component because the phase of this echo has the same properties as that of the double-quantum (DQ) signal. Using a partition method and computer simulation can eliminate the magnetization components of the intersequence stimulated echo [22]. The DQ filter pulse sequence with non-refocused preparation time $(\tau \mathrm{P})$ and evolution time $(\tau \mathrm{E})$ may be expressed as: $\left(\theta_{1}, \varphi_{1}\right)-\tau \mathrm{P}-\left(\theta_{2}, \varphi_{2}\right)-\tau \mathrm{E}-\left(\theta_{3} \varphi_{3}\right)-\operatorname{Acq}(\mathrm{t} 2, \varphi \mathrm{R})$, where $\varphi_{\mathrm{n}}$ denotes the phase angle of each RF pulse with the flip angle $\varphi_{\mathrm{n}} ; \mathrm{t} 2$ is acquisition or detection time and $\varphi R$ is receiver phase.

\subsection{Multiple Quantum Filters of Arbitrary Phases of Na-23 Nuclei}

These Triple Quantum filtered data acquisition, uses the pulse sequence with arbitrary phase values: $90^{\circ}{ }_{(\theta 1)}-\mathrm{t} / 2$ $-180^{\circ}{ }_{(\theta 2)}-\mathrm{t} / 2-90^{\circ}{ }_{(\theta 3)}-\delta-90^{\circ}{ }_{(\theta 4)}-\operatorname{acq}_{(\theta 5)}(\mathrm{T})$, where $\delta$ is evolution time, $\mathrm{t}$ is the acquisition time, $\theta 1, \theta 2, \theta 3, \theta 4$, and $\theta 5$ are the phase values. Multiple-quantum-filtered (MQF) Na-23 NMR spectroscopy may provide increased sensitivity in detecting IC Na and thus offers the possibility of monitoring changes in IC $\mathrm{Na}$ content without $\mathrm{SR}$. The amplitude of the MQF spectrum is determined by several factors such as transverse relaxation times, creation times, and the amount of $\mathrm{Na}$ that exhibits MQ coherence. By the behavior of relaxation and greater IC-Na MQ coherence, it is possible to have MQF spectrum with intracellular sodium to measure IC-Na content [23].

\subsection{Spiral Cardiac Respiratory Gate Multi-Shot Functional MRI as Possibility}

Physiological fluctuations (possibly by sodium) are known to be a major contribution to noise in fMRI data. Cardiac noise adds noise to both the magnitude and phase of the image in regions localized near vessels and ventricles. Another factor, respiratory motion, has both global changes in the phase of image and localized variations in the image magnitude, particularly near ventricles. Recently, an empirical model was used to capture the observed features of physiological noise in fMRI data to reduce intracellular ionic noise in fMRI data using a 3-D cylindrical-stack spiral pulse sequence [24]. Briefly, spiral waveforms of sequence were applied on the $\mathrm{x}$ and $\mathrm{y}$ gradients. The waveforms were designed get low slew rate limited except for the first few points, and their maximum amplitude was $0.75 \mathrm{G} / \mathrm{cm}$ to give a $3.8 \mathrm{x}$ $3.8 \mathrm{~mm}$ nominal in-plane resolution. Six interleaves were measured by adjusting the spiral waveforms (multiplying the $\mathrm{x}$ and $\mathrm{y}$ gradients by coefficients of a rotation matrix) to effectively rotate the k-space trajectory in the $\mathrm{k}_{\mathrm{x}}-\mathrm{k}_{\mathrm{y}}$ plane. At the end of each TR interval, crusher gradients were used to minimize spillover of the FID signal into subsequent intervals. Their amplitudes were set to generate about $3 \pi$ dephasing over each voxel dimension of covered volume of interest. It results in a "stacked spirals" k-space acquisition scheme. With the spiral acquisition, all gradient moments, $\mathrm{Mn}(\mathrm{t})$, can be shown as Eq.10:

$$
M_{n}(t)=\int G_{(\mathrm{t})} t_{\mathrm{n}} d t
$$

with $\mathrm{t}=0$ at the center of the RF pulse, are zero at the center of k-space $\left(t=t_{c}\right)$.

The zero $^{\text {th }}\left[\mathrm{M}_{0}(\mathrm{t})\right]$ and first $\left[\mathrm{M}_{1}(\mathrm{t})\right]$ order moments of the slab select null at gradient at $t=t_{c}$ by using two additional gradient phases after the slice selection lobe. Furthermore, the magnetization were nulled at $\mathrm{t}=\mathrm{TR}$ i.e., $M_{0}(t)$ for the phase encode gradient and $M_{0}(t)$ and $M_{1}(t)$ for the spiral waveforms. These spiral waveforms are made of trapezoid waveforms at amplitude with minimum gradient moments. In addition, a phase encoding scheme is inverted and quadratic RF phase modulation is performed to minimize stimulated echoes. Additional gradient pulses can minimize stimulated echoes after acquisition. Another good practice to decrease the scan time is inserting extra 'dummy views' to avoid extra acquisitions when the physiological phase corresponds to the view far away from the actual view to acquire, although these 'dummy views' reduce fMRI temporal resolution and lead to improper assessment of functional activity \& physiological variance. Cardiac ordering shows greater utility due to shorter cardiac periods. It can reduce inter-image variance by using k-space postprocessing techniques and may enhance sodium contrast. This technique is very promising but longer scan times are required.

\section{CLINICAL SODIUM MRI TRIALS}

Sodium MRI can be used in anatomical imaging applications in brain, tumors and cardiac cycle (25-29). Special attention was focused on achieving intracellular sodium images. Triple Quantum filter in spin echo acquisition mode and inversion recovery methods are considered useful for it without using shift contrast agents. However, the triple-quantum filtered technique requires a very long repetition time. Inversion recovery technique suffers from poor nulling and poor suppression of extracellular or intracellular sodium population (30-31). Moreover, for imaging applications with the resolutions of 64 or 128 pixels, physiological noise corrupt even better regions without variation. These are referred to as 'ghosts', and may be shifted away from the area of in- 
terest or avoided by using the 'view ordering' technique. It was done by sorting the views for which physiological period $\mathrm{T}=\mathrm{T}_{0}(\alpha=1)$ 'low frequency sort mode' or $\mathrm{T}=\mathrm{T}_{0}$ $x$ N/2 'high frequency sort mode', where $\alpha=\mathrm{N} / 2$, $\mathrm{N}=$ total number of views). For cardiac sodium MRI data acquisition in real time, the physiological phase and corresponding view are obtained again and again till all views are expended. Physiological ghosts, phase variation is cause of further inter-image fluctuations in cardiac MRI time course and affect activation related changes in the acquired signal.

Intracellular sodium concentration measurement: Several attempts to measure the intracellular sodium concentration including sodium flame photometry and ion electrodes in serum; ratiometric, electrom beam CT, atomic force (AFM) spectroscopy in tumors; and sodium MRS in myocardium suggested the role of ATP energy and $\mathrm{Na}+/ \mathrm{K}+$ pump in ischemia \& tumor apoptosis. Therapies that alter tumor ion homeostasis or affect/ destroy tumor cell membrane integrity are likely to generate changes that are observable with ${ }^{23} \mathrm{Na} \mathrm{MR}$ imaging and sodium concentration measurements [29,31]. For different methods, the concentration of intracellular sodium can be measured based on NMR peak areas by subtraction or magnetization ratio and intracellular/extracellular volumes as following:

For MQ method,

$[N a]_{i(M Q}=\left\{1-\frac{[y] M_{M 0(x)}}{[x] M_{M 0, v(y)}}\right\}\left\{V_{R} / V_{i n}\right\}[N a]_{R} \sim M_{0, v M(x)}$

where $[\mathrm{x}]$ and $[\mathrm{y}]$ denote the corresponding tissue and standard phantom intracellular sodium concentrations. $\mathrm{K}$ is a constant equal to $\mathrm{M}^{\mathrm{M} 0, \mathrm{v}(\mathrm{y})} /[\mathrm{y}] . \mathrm{V}_{\mathrm{R}} / \mathrm{V}_{\text {in }}$ is the ratio of reference and intracellular volumes in tissue.

For DQ method,

$$
\left.\left[\mathrm{Na}_{\mathrm{i}+}\right]^{\mathrm{DQ}}=\left\{\left(\mathrm{A}_{\mathrm{in}} / \mathrm{A}_{\text {ref }}\right) \text {-(intercept }\right)\right\} /(\text { slope })
$$

where $A_{\text {in }}$ and $A_{\text {ref }}$ represent areas under tissue and phantom intracellular sodium peaks.

For SQ method,

$$
\mathrm{IC}[\mathrm{Na}]=\left\{\mathrm{A}_{\text {in }} / \mathrm{A}_{\text {out }}\right\}\left\{\mathrm{V}_{\text {out }} / \mathrm{V}_{\text {in }}\right\}\{\mathrm{EC}[\mathrm{Na}]\}
$$

$\mathrm{A}_{\text {in }}$ and $\mathrm{A}_{\text {out }}$ are areas of the $\mathrm{SQ}{ }^{23} \mathrm{Na}$ NMR peaks for IC and EC spaces; EC [Na] is extracellular $\mathrm{Na}$ concentration.

With these measurements, changes can be observed much earlier than with the effects of anatomic remodeling. ${ }^{1} \mathrm{H}$ MR imaging FLAIR sequence, use of MR contrast agents, and T2-weighted imaging along with ${ }^{23} \mathrm{Na}$ MR imaging may improve tumor visualization of a necrotic core or proliferating zones by multiparametric analysis methods. Other approaches of enhancement of $3 \mathrm{D}{ }^{23} \mathrm{Na}$ images signal-to noise ratio depend on receivers and coils and the twisted-projection imaging pulse sequence [32].
The sodium concentration may be measured by placing a phantom next to tumor or animal during MR imaging. The mean sodium concentration per kilogram wet body weight in the region of interest will be:

$$
[N a]_{k}=\frac{\frac{I_{k}}{R_{k} S F_{k}}-b}{a D}
$$

The coefficients a and $\mathrm{b}$ were calculated from the sodium concentrations $\mathrm{C}_{1}$ and $\mathrm{C}_{2}$ and signal intensities $\mathrm{I}_{1}$ and $\mathrm{I}_{2}$ of the two phantoms as

$$
a=\frac{\frac{I_{2}}{R_{k} S F_{2}}-\frac{I_{1}}{R_{1} S F_{1}}}{C_{2}-C_{1}}
$$

and

$$
b=\frac{I_{2}}{R_{2} S F_{2}}-a C_{2}
$$

where $R_{1}$ and $R_{2}$ are sensitivity factors.

In the following section, we describe our experiments in support of increased sodium in malignancy and association with apoptosis in breast tumors and epithelial growth factor in breast isolated cancer cells. For simplicity, we introduce readers to the biochemical basis of increased sodium in tumor cells. The low sodium-hydrogen exchange kinetics by slow sodium-hydrogen transporter is center point. In malignant tumor cells, acidic $\mathrm{pH}$ enhances the intracellular sodium that impairs both the sodium-hydrogen transporter ability and $\mathrm{Na}^{+} / \mathrm{K}^{+}$ pump resulting in reduced $\left[\mathrm{Na}^{+} / \mathrm{K}^{+}\right]$ATPase enzyme to release sodium (high intracellular sodium concentration inside cells) from mitochondrial oxidation which also triggers cells to slow down apoptosis or other associated epithelial growth factors [high intracellular $\mathrm{Na}$ with low apoptosis and EGF]. The low concentration of intracellular sodium is sensitive to any malignancy change and serves as a diagnostic rapid MRI imaging assay to test drugs.

\subsection{Chemosensitivity Rapid Assays Using Sodium MRI}

Very limited studies reported the value of intracellular sodium in a chemosensitivity assessment by cell proliferation and apoptosis in prostate and breast tumors. However, its applications are expanding to glioma, cartilage, and liver [32,33]. A technical advance was reported using an inversion recovery pulse sequence and optimization of inversion times to achieve intracellular sodium images in less time. Its application was applied in texotere chemosensitivity response to PC 3-induced mouse prostate and $\mathrm{MCF} 7$-induced rat breast tumors as shown in Figure 3. The enhanced contrast of increased intracellular sodium MRI signal was correlated with histopathology characterization of tumor tissues [34,35, 


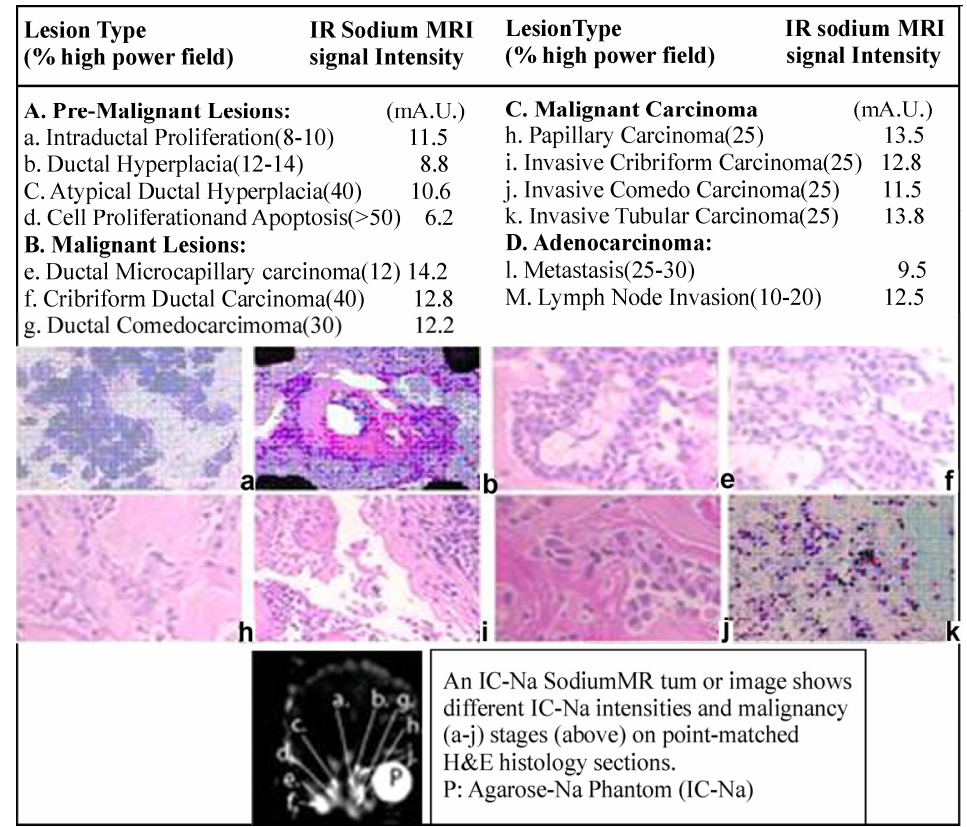

Figure 3. Inversion recovery pulse sequence using optimized inversion time generated high resolution intracellular sodium images of tumor (right on the phantom image) in each panel. On right, a tumor image is enlarged to show regional heterogeneity of MCF 7 induced breast tumor in rat. $\mathrm{P}$ represents image of agarose-sodium phantom and alphabets at different points indicate different stages of tumor growing cells to correlate with histology as shown in table to highlight the accuracy of sodium MR signal intensity and tumor pathology staging [36].

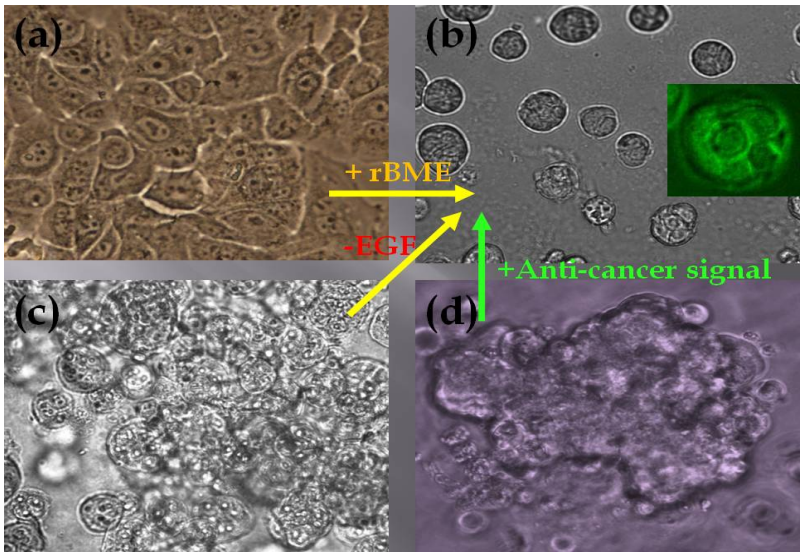

Figure 4. A Comparison between normal and malignant human breast epithelial cells (grown in a 2D monolayer and 3D culture matrix) shows a role of EGF in luminal structure formation. Normal human breast epithelial (NHBE) cells (grown in the 3D culture matrix) form a luminal structure of normal phenotype. (a) Normal human breast epithelial cells in monolayer without $\mathrm{rBME}$ (2D monolayer) with normal EGF, (b) NHBE cells in rBME (3D matrix) with reduced EGF, (c) NHBE cells in rBME (3D) with normal EGF, (d) Malignant human breast epithelial cells (HTB-132 obtained from ATCC) in $\mathrm{rBME}(3 \mathrm{D})$ with reduced EGF. Notice the role of FGF and the rBME matrix used in association with malignant characteristic of cells.
36]. However, the exact cause of increased TSC is not known. In another set of breast cancer culture cells, we observed the reduced EGF in tissues sensitive to sodium ion homeostasis and metabolic integrity.

\subsection{Correlation between Intracellular Sodium Signal and Malignancy}

Elevated TSC in breast lesions measured by non-invasive $23 \mathrm{Na}$ MRI appears to be an indicator at the cellular level associated with malignancy. This Na-23 MRI method may have potential to improve the specificity of breast MRI with only a modest increase in scan time per patient [37]. Several physiological and biochemical changes associated with proliferating malignant tumors may cause an increase in total tissue sodium concentration (TSC) as a result of impaired sodium-hydrogen transporter and $[\mathrm{Na} / \mathrm{K}]$ pump activity resulting in reduced EGF (Figure 5).

Normal and malignant epithelial ductal cells were grown on a reconstituted basement membrane extract (rBME). In the event of the correct signaling from growth factor and extra-cellular matrix proteins, isolated human breast epithelial (HBE) cells formed their original phenotype in vivo. HBE cells in the absence of reconstituted basement membrane extract (rBME) failed to assemble organized structures, and arrested growth when 
Sodium

Null Points (A) and (B) (TQ, DQ and MQ filters show difference)

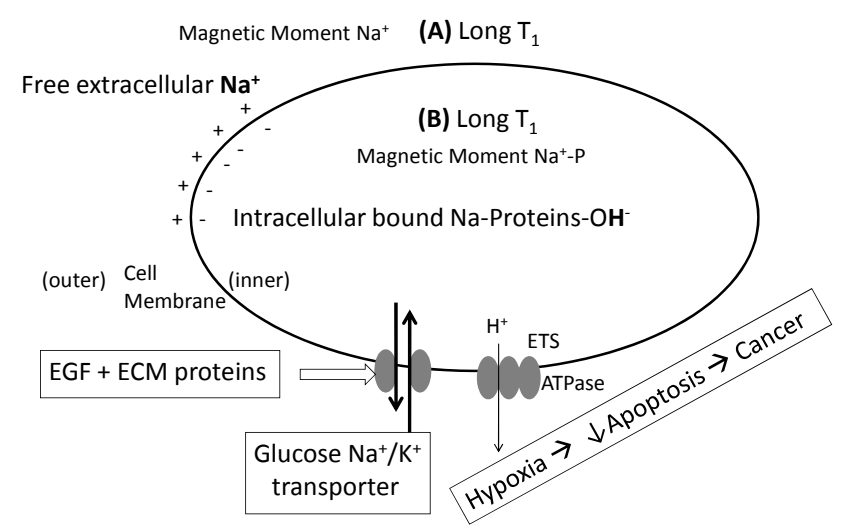

Figure 5. This schematic description represents the origin of intracellular sodium across the membrane and different null points of sodium inside and outside generate contrast due to different longitudinal relaxation constants as basis of sodium MRI. The increased intracellular sodium leakage out of cells is associated with hypoxia and apoptosis in cancer. EGF: Epidermal Growth Factor, ECM: Extracellular matrix, and ETS: Electron Transfort System.

they reached confluence. HBE cells on-top of rBME could display an acinar structure with a dead luminal space in the absence of epidermal growth factors (EGF) (Figure 4(b)). This simulated the ductal structures of breast epithelial cells found in vivo. Under the same condition, carcinoma cells exhibited colony overgrowth, luminal filling, and loss of intracellular sodium (Figure 4(d)). These changes eventually reduce the resistance against apoptosis and enhance the cell proliferation resulting in severe morphological deformities visible by microscopy and imaging as earlier reported elsewhere [37]. It was observed that EGF disrupted the formation of luminal structures of HBE either in monolayer or in 3 dimensional cell cultures with rBME. It may be attributed with the possibility if luminal structures bound with sodium get free by the disruption after adding EGF. When EGF was reduced or removed from the cultures, normal phenotypes (e.g. luminal structure and uniformed size) of human breast epithelial cell were obtained. With the variation of the time to reduce EGF, we could generate different sizes of luminal structure of $\mathrm{HBE}$

\subsection{Limitations of Sodium Contrast and Sensitivity}

Due to high concentrations of extracellular sodium, its suppression by using inversion recovery pulses makes it difficult to get absolute intracellular sodium images. However, intracellular sodium images may be indicators of edema, interstitial space, cell proliferation, malignancy, and hypoxia as criteria to divide tumor dormancy, slow-growing, and fast growing regions. Newer techniques without the use of shift reagents using high reso- lution $\mathrm{RF}$ coils generate $3 \mathrm{D}^{23} \mathrm{Na}$ images with high signal-to noise ratio in less than 15 minutes, permitting use of combined ${ }^{23} \mathrm{Na}$ and ${ }^{1} \mathrm{H}$ MR imaging protocols with total examination times of about 45 minutes. Moreover, sodium imaging in myocardium and cardiac ischemia is well investigated as a clinical tool.

\section{CONCLUSIONS}

A present state-of-art for sodium MR imaging is presented with a focus on quantum filters in pulse sequences and their variants in order to acquire intracellular sodium images with hands-on software pulse sequence design and MRI physics principles. Triple quantum filtering scheme and non-invasive inversion recovery fast spin-echo pulse sequences are described for distinct intracellular sodium MR images. Our experiments on breast tumors and isolated culture cells indicated the association of malignancy with increased intracellular sodium and reduced apoptosis and reduced EGF in cells with possibility of other growth factors involved.

\section{ACKNOWLEDGEMENTS}

The present work was supported by the grant GIA 0086 funded by Aventis Pharmaceutical Company Inc. for design and development of sodium MRI technique. Partly the work was presented at workshop ISMRM: Minimization of data acquisition with maximum outcome in 2002. The study for the normal and malignant breast epithelial cell culture was supported by NIH (1 R21 CA 131798-01A1).

\section{REFERENCES}

[1] I. L. Cameron, N. K. Smith, T. B. Pool, and R. L. Sparks, 
(1980) Intracellular concentration of sodium and other elements as related to mutagenesis and oncogenesis in vivo, Cancer Res., 40(5), 1493-500.

[2] A. Amidsen and M. Schou, (1968) Lithium and the transfer rate of sodium across the blood-brain barrier, Psychopharmacologia, 12(3), 236-238.

[3] B. J. Carroll, L. Steven, R. A. Pope, and B. Davies, (1969) Sodium transfer from plasma to CSF in severe depressive illness, Arch. Gen. Psychiatry, 21(1), 77-81.

[4] M. E. Moseley, W. M. Chew, M. C. Nishimura, T. L. Richards, J. Murphy-Boesch, G. B. Young, T. M. Marschner, L. H. Pitts, and T. L. James, (1985) In vivo sodium-23 magnetic resonance surface coil imaging: Observing experimental cerebral ischemia in the rat, Magn. Reson. Imaging, 3(4), 383-387.

[5] W. H. Perman, P. A. Turski, L. W., Houston, G. H. Glover, and C. E. Hayes, (1986) Methodology of in vivo human sodium MR imaging at $1.5 \mathrm{~T}$, Radiology, 160(3), 811-820.

[6] S. S. Winkler, D. M. Thomasson, K. Sherwood, and W. H. Perman, (1989) Regional T2 and sodium concentration estimates in the normal human brain by sodium-23 MR imaging at 1.5 T, J. Comput. Assist. Tomogr., 13(4), 561566.

[7] J. M. Dizon, J. S. Tauskela, D. Wise, D. Burkhoff, P. J. Cannon, and J. Katz, (1996) Evaluation of triplequantum-filtered 23Na NMR in monitoring of Intracellular $\mathrm{Na}$ content in the perfused rat heart: comparison of intra- and extracellular transverse relaxation and spectral amplitudes, Magn. Reson. Med., 35(3), 336-345.

[8] K. J. Jung and J. Katz, (1996) Chemical-shift-selective acquisition of multiple-quantum-filtered $23 \mathrm{Na}$ signal, J. Magn. Reson. B., 112(3), 214-227.

[9] P. G. Morris, (1986) Nuclear magnetic resonance imaging in medicine and biology, Clarendon Press, Oxford, England, 123.

[10] S. W. Lee, S. K. Hilal, and Z. H. Cho, (1986) A multinuclear magnetic resonance imaging technique-simultaneous proton and sodium imaging, Magn. Reson. Imaging, 4(4), 343-350.

[11] P. J. Cannon, A. A. Maudsley, S. K. Hilal, H. E. Simon, and F. Cassidy, (1986) Sodium nuclear magnetic resonance imaging of myocardial tissue of dogs after coronary artery occlusion and reperfusion, J. Am. Coll. Cardiol., 7(3), 573-579.

[12] C. T. Moonen, S. E. Anderson, and S. Unger, (1987) $23 \mathrm{Na}$ rotating frame imaging in the perfused rabbit heart using separate transmitter and receiver coils, Magn. Reson. Med., 5(3), 296-301.

[13] R. Ouwerkerk, K. B. Bleich, J. S. Gillen, M. G. Pomper, and P. A. Bottomley, (2003) Tissue sodium concentration in human brain tumors as measured with $23 \mathrm{Na} \mathrm{MR}$ imaging. Radiology, 227(2), 529-3.

[14] F. E. Boada, G. X. Shen, S. Y. Chang, and K. R. Thulborn, (1997) Spectrally weighted twisted projection imaging: reducing T2 signal attenuation effects in fast three-dimensional sodium imaging, Magn. Reson. Med., 38(6), $1022-1028$

[15] I. Hancu, F. E. Boada, and G. X. Shen, (1999) Threedimensional triple-quantum-filtered (23) Na imaging of in vivo human brain, Magn. Reson. Med., 42(6), 11461154.
[16] A. Borthakur, I. Hancu, F. E. Boada, G. X. Shen, E. M. Shapiro, and R. Reddy, (1999) In vivo triple quantum filtered twisted projection sodium MRI of human articular cartilage, J. Magn. Reson., 141(2), 286-290.

[17] K. J. Jung, P. J. Cannon, and J. Katz, (1997) Simultaneous acquisition of quadrupolar order and doublequantum 23Na signals, J. Magn. Reson., 129(2), 130-133.

[18] L. M. Boxt, D. Hsu, J. Katz, P. Detweiler, S. McLaughlin, T. J. Kolb, and H. M. Spotnitz, (1993) Estimation of myocardial water content using transverse relaxation time from dual spin-echo magnetic resonance imaging, Magn. Reson. Imaging, 11(3), 375-383.

[19] G. X. Shen, J. F. Wu, F. E. Boada, and K. R. Thulborn, (1999) Experimentally verified, theoretical design of dual-tuned, low-pass birdcage radiofrequency resonators for magnetic resonance imaging and magnetic resonance spectroscopy of human brain at 3.0 Tesla, Magn. Reson. Med., 41(2), 68-275.

[20] K. J. Jung, J. Katz, L. M. Box, S. K. Hilal, and Z. H. Cho, (1995) Breakthrough of single-quantum coherence and its elimination in double-quantum filtering, J. Magn. Reson. B., 107(3), 235-241.

[21] K. J. Jung, J. S. Tauskela, and J. Katz, (1996) New double-quantum filtering schemes, J. Magn. Reson. B., 112(2), 103-110.

[22] K. J. Jung and J. Katz, (1997) Mathematical analysis of generation and elimination of intersequence stimulated echo in double-quantum filtering, J. Magn. Reson., 124 (1), 232-236.

[23] J. S. Tauskela, J. M. Dizon, J. Whang, and J. Katz, (1997) Evaluation of multiple-quantum-filtered $23 \mathrm{Na} \mathrm{NMR}$ in monitoring intracellular $\mathrm{Na}$ content in the isolated perfused rat heart in the absence of a hemical-shift reagent, J. Magn. Reson., 127(1), 115-127.

[24] V. A. Stenger, S. Peltier, F. E. Boada, and D. C. Noll, (1999) 3D spiral cardiac/respiratory ordered fMRI data acquisition at 3 Tesla, Magn. Reson. Med., 41(5), 983991.

[25] H. Serrai, A. Borthakur, L. Senhadji, and R. Reddy, (2000) Bansal, N. Time-domain quantification of multiple-quantum-filtered (23) Na signal using continuous wavelet transform analysis, J. Magn. Reson. 142(2), 341-347.

[26] J. B. Ra, S. K. Hilal, C. H. Oh, and I. K. Mun, (1988) In vivo magnetic resonance imaging of sodium in the human body. Magn Reson Med., 7(1), 11-22.

[27] S. K. Hilal, A. A. Maudsley, J. B. Ra, H. E. Simon, P. Roschmann, S. Wittekoek, Z. H. Cho, and S. K. Mun, (1985) In vivo NMR imaging of sodium-23 in the human head, J Comput Assist Tomogr, 9 (1), 1-7.

[28] T. Hashimoto, H. Ikehira, H. Fukuda, A. Yamaura, O. Watanabe, Y. Tateno, R. Tanaka, and H. E. Simon, (1991) In vivo sodium-23 MRI in brain tumors: Evaluation of preliminary clinical experience, Am J Physiol Imaging, 6(2), 74-80.

[29] K. L. Allen, A. L. Busza, S. R. Williams, and S. C. Williams (1994) Early changes in cerebral sodium distribution following ischaemia monitored by $23 \mathrm{Na}$ magnetic resonance imaging, Magn Reson Imaging, 12(6), 895900.

[30] R. Sharma and R. P. Kline, (2004) Chemosensitivity assay in mice prostate tumor: Preliminary report of flow 
cytometry, DNA fragmentation, ion ratiometric methods of anti-neoplastic drug monitoring. Cancer Cell International Cancer Cell International, 4(3).

[31] R. P. Kline, E. X. Wu, D. P. Petrylak, M. Szabolcs, P. O. Alderson, M. L. Weisfeldt, P. Cannon, and J. Katz, (2000) Rapid in vivo monitoring of chemotherapeutic response using weighted sodium magnetic resonance imaging, Clin Cancer Res., 6(6), 2146-56.

[32] P. M. Winter, V. Seshan, J. D. Makos, A. D. Sherry, C. R. Malloy, and N. Bansal, (1998) Quantitation of intracellular $\left[\mathrm{Na}^{+}\right]$in vivo by using TmDOTP5-as an NMR shift reagent and extracellular marker, J Appl Physiol. 85(5), $1806-12$.

[33] P. M. Winter, H. Poptani, and N. Bansal, (2001) Effects of chemotherapy by 1,3-bis(2-chloroethyl)-1-nitrosourea on single-quantum- and triple-quantum-filtered $23 \mathrm{Na}$ and 31P nuclear magnetic resonance of the subcutaneously implanted 9L glioma, Cancer Res., 61(5).

[34] J. M. Colet, N. Bansal, C. R. Malloy, and A. D. Sherry,
(1999) Multiple quantum filtered 23Na NMR spectroscopy of the isolated, perfused rat liver, Magn Reson Med. 41(6), 1127-35.

[35] R. Sharma, R. P. Kline, E. X. Wu, and J. K. Katz, (2005) Rapid in vivo Taxotere quantitative chemosensitivity response by 4.23 Tesla sodium MRI and histo-immunostaining features in N-Methyl-N-Nitrosourea induced breast tumors in rats, Cancer Cell International, 5, 26.

[36] R. Sharma, (2008) Extended expression for transverse magnetization using four pulse sequence to construct double quantum filter of arbitrary phases for spin $3 / 2$ sodium nuclei, International J. Computer Research, 16(4), 371-388.

[37] R. Ouwerkerk, M. A. Jacobs, K. J. Macura, A. C. Wolff, V. Stearns, and S. D. Mezban, N. F. Khouri, D. A. Bluemke, and P. A. Bottomley, (2007) Elevated tissue sodium concentration in malignant breast lesions detected with non-invasive ${ }^{23} \mathrm{Na} \mathrm{MRI}$, Breast Cancer Research and Treatment, 106(2), 151-60. 